\title{
AVALIAÇÃO SENSORIAL E MAPA DE PREFERÊNCIA INTERNO DE MARCAS COMERCIAIS DE REFRIGERANTE SABOR GUARANÁ
}

\author{
JOÃO DE DEUS SOUZA CARNEIRO * \\ RONIELLI CARDOSO REIS ** \\ VALÉRIA PAULA RODRIGUES MINIM *** \\ JOEL CAMILO SOUZA CARNEIRO **** \\ ADAIR JOSÉ REGAZZI ***** \\ JANE SÉLIA DOS REIS COIMBRA \\ LUIS ANTÔNIO MINIM
}

O objetivo deste trabalho foi comparar sensorialmente sete marcas de refrigerante sabor guaraná, sendo seis produzidas por pequenas empresas e uma por empresa líder de mercado. A Análise Descritiva Quantitativa (ADQ) foi utilizada para descrever e quantificar os atributos sensoriais das amostras. A aceitabilidade sensorial das marcas de refrigerante foi determinada mediante escala hedônica e os resultados avaliados pela Análise de Componentes Principais, Mapa de Preferência Interno e ANOVA. A maioria dos consumidores preferiu o refrigerante da marca líder de mercado, que apresentou os atributos sabor e aroma característico em maior intensidade e sabor e aroma tutti-fruti em menor intensidade.

PALAVRAS-CHAVE: GUARANÁ; REFRIGERANTES-ANÁLISE SENSORIAL; MAPA DE PREFERÊNCIA.

* $\quad$ Mestre em Ciência e Tecnologia de Alimentos, Departamento de Tecnologia de Alimentos (DTA), Universidade Federal de Viçosa (UFV), Viçosa, MG (e-mail: joaodc@hotmail.com).

** Mestre em Ciência e Tecnologia de Alimentos, DTA, UFV, Viçosa, MG (e-mail: roniellireis@hotmail.com).

*** Doutora em Ciência de Alimentos, DTA, UFV, Viçosa, MG. (e-mail: vprm@ufv.br).

**** Mestre em Ciência e Tecnologia de Alimentos, DTA, UFV, Viçosa, MG (e-mail: jcarneiro@hotmail.com).

***** Doutor em Estatística e Experimentação Agronômica, Departamento de Informática, UFV, Viçosa, MG (e-mail: adairreg@ufv.br).

****** Doutor em Engenharia de Alimentos, DTA, UFV, Viçosa, MG (e-mail: jcoimbra@ufv.br).

****** Doutor em Engenharia Química, DTA, UFV, Viçosa, MG (e-mail: Iminim@ufv.br). 


\section{INTRODUÇÃO}

Refrigerantes são bebidas não-alcoólicas, gaseificadas com dióxido de carbono, obtidas pela dissolução em água potável de açúcares, suco de frutas, extrato de sementes e outras partes inócuas de vegetais, bem como substâncias permitidas pela legislação vigente (BRASIL, 2001).

O mercado brasileiro de refrigerantes tornou-se o terceiro em nível mundial, com consumo de 11 bilhões de litros em 1998. Apresenta amplo potencial de crescimento em função do baixo volume de consumo per capta, quando comparado aos Estados Unidos da América e México, países com as maiores demandas de refrigerante do mundo (SANTOS e AZEVEDO, 1999).

O mercado de refrigerantes vem passando por intensa reestruturação, bastante distinta da concentração experimentada pelo setor alimentício na década de 90 . Tem sido observado intenso crescimento de pequenas empresas (atuação predominantemente regional), constituindo processo de desconcentração (SANTOS e AZEVEDO, 1999).

No período entre 1990 e 1999, o aumento na participação das pequenas empresas cresceu de 9,2 para 33\% nesse mercado (SEAE, 2000). Tal crescimento e a possibilidade de faturamento garantido, baseado na oferta com preços reduzidos, estimulou o surgimento de novas marcas.

A estabilidade econômica fortaleceu as marcas regionais, assim como a liberação de preços e o acesso a novas tecnologias. Mais de 200 pequenas empresas produtoras de refrigerante surgiram nos últimos três anos, respondendo pelo investimento de 500 milhões de reais (FERNANDES, 2000). Outro fator que proporcionou o crescimento das pequenas e médias empresas de refrigerantes foi o aparecimento das embalagens de polietileno tereftalato (PET). Em 1999, segundo SANTOS e AZEVEDO, o Brasil já contava com cerca de 700 empresas fabricantes de refrigerantes, a maioria criada após a introdução desse tipo de embalagem. Entretanto, ainda não existe nenhum estudo científico a respeito da qualidade sensorial desses produtos. 
O objetivo deste trabalho foi comparar os atributos sensoriais de sete marcas de refrigerante sabor guaraná, sendo seis produzidas por pequenas empresas e uma por empresa líder de mercado.

\section{MATERIAL E MÉTODOS}

\subsection{MATERIAL}

Os refrigerantes avaliados foram adquiridos no comércio de Viçosa/ MG (Brasil), sendo as amostras P1, P2, P3, P4, P5 e P6 produzidas por pequenas empresas e a ML por empresa líder de mercado.

\subsection{ANÁLISE DESCRITIVA QUANTITATIVA (ADQ)}

O método empregado foi adaptado da Análise Descritiva Quantitativa, desenvolvida por STONE et al. (1974), que permite descrever as principais características dos alimentos (aparência, aroma, textura e sabor), além de medir a intensidade das sensações percebidas (STONE e SIDEL, 1993).

\subsubsection{Condições do Teste}

As avaliações ocorreram no Laboratório de Análise Sensorial do Departamento de Tecnologia de Alimentos da Universidade Federal de Viçosa, entre 15h30min e 18h. A aparência foi avaliada sob luz natural, sendo o aroma e o sabor avaliados em cabines individuais com luz vermelha. As discussões abertas entre os participantes da equipe sensorial para obtenção da lista de atributos e treinamento foram realizadas em mesa redonda.

\subsubsection{Apresentação das Amostras}

As amostras foram codificadas com números de três dígitos e servidas (temperatura de $5^{\circ} \mathrm{C}$ ) aos julgadores, em copos descartáveis $(50 \mathrm{~mL}$ ) para avaliação do aroma e do sabor e em copos de vidro transparente (200 mL) para avaliação da aparência. 


\subsubsection{Julgadores}

A equipe de julgadores foi recrutada por meio de questionário, distribuído a 40 candidatos, conforme a disponibilidade de tempo, consumo de refrigerante e habilidade para utilizar a escala e definir termos descritivos. Na pré-seleção dos candidatos foi aplicada uma série de quatro testes triangulares, utilizando-se duas marcas de refrigerante (ML e P3). Foram selecionados os provadores que obtiveram porcentagem de acerto maior ou igual a $75 \%$.

\subsubsection{Levantamento de Atributos e Treinamento dos Julgadores}

Para levantamento dos atributos sensoriais foi utilizado o método rede (MOSKOWITZ, 1983). As sete amostras de refrigerante foram apresentadas aos julgadores duas a duas, formando todos os pares possíveis, visando à identificação das similaridades e diferenças entre cada par de amostras. Em seguida, os julgadores discutiram os termos levantados a fim de eliminar redundâncias ou termos poucos citados, sendo selecionados de forma consensual os termos que melhor descreveram as diferenças e similaridades entre as amostras. Durante essa fase do trabalho, a equipe também sugeriu amostras de referência para cada atributo levantado visando o treinamento dos julgadores e a padronização na utilização de cada termo.

Após a realização de várias sessões foram obtidos os termos descritivos das amostras, a ficha de avaliação, a lista de referência e a definição de cada termo descritivo, por consenso da equipe sensorial.

A ficha de avaliação continha escala não-estruturada para cada termo descritivo levantado. A escala, composta por linha de nove centímetros, apresentava expressões quantitativas (pontos-âncoras) nas extremidades esquerda e direita com os termos "pouco", "ausente", "fraco", "muito" e "forte", respectivamente. Os julgadores, mediante traço vertical na escala, escolheram a posição de sua avaliação para cada termo descritivo. Os valores numéricos para cada atributo foram obtidos medindo-se a distância entre o ponto-âncora da extremidade esquerda e o traço vertical feito pelo provador, com o auxílio de uma régua. 


\subsubsection{Testes Preliminares e Seleção de Julgadores}

Após a etapa de treinamento foram realizados testes preliminares para avaliar o desempenho dos julgadores, quanto à repetibilidade e poder de discriminação. Para tanto, os julgadores avaliaram três amostras (ML, P1 e P4), na mesma seção, com três repetições em relação a cada atributo levantado. Para a seleção dos julgadores efetuou-se análise de variância (ANOVA) dos dados, por julgador e para cada atributo sensorial, cujo modelo estatístico incluiu duas fontes de variação além do erro (repetições e amostras). Os julgadores foram selecionados com base em sua capacidade discriminatória ( $\mathrm{pF}_{\text {amostra }}$ $\leq 0,50)$ e repetibilidade $\left(\mathrm{pF}_{\text {repetição }}>0,05\right)$, conforme sugerido pela ASTM (1976) e por DAMÁSIO e COSTELL (1991).

\subsubsection{Avaliação das Amostras e Análise dos Resultados}

Utilizando-se os atributos levantados, nove julgadores selecionados e treinados $\left(\mathrm{pF}_{\text {amostra }} \leq 0,50\right.$ e $\left.\mathrm{pF}_{\text {repetição }}>0,05\right)$ avaliaram as sete amostras, com três repetições. Optou-se pelo delineamento em blocos incompletos casualizados (COCHRAM e COX, 1981), com os seguintes parâmetros: $\mathrm{t}=$ número total de amostras avaliadas $=7 ; \mathrm{k}=$ número de unidades por blocos $=3 ; r=$ número de repetições $=3 ; b=$ número de blocos $=7$.

Os resultados foram compilados em tabela de dupla entrada (julgadores e amostra). Em seguida, os dados foram submetidos à Análise de Variância (ANOVA) e Análise de Componentes Principais. Todas as análises foram realizadas com o programa SAS (1999), versão 8.0, licenciado para UFV.

\subsection{TESTE DE ACEITAÇÃO}

Cinqüenta consumidores de refrigerante avaliaram sete amostras de guaraná, servidas de forma monádica. Utilizou-se a escala hedônica estruturada de nove pontos (Figura 1) para verificar o quanto gostaram ou desgostaram de cada marca de refrigerante.

Os resultados conseguidos foram utilizados para obtenção do mapa de preferência interno. 
FIGURA 1 - FICHA DE AVALIAÇÃO USADA PARA O TESTE DE ACEITAÇÃO

\begin{tabular}{|l} 
ESCALA HED NICA \\
IDADE: SEXO: ( ) M ( ) F \\
Por favor, prove a amostra utilizando a escala abaixo e identifique o quanto \\
vocCEgostou ou desgostou do produto. Marque a posi a o da escala que melhor \\
reflita o seu julgamento. \\
( ) Gostei extremamente \\
( ) Gostei muito digo da amostra: \\
( ) Gostei moderadamente \\
( ) Gostei ligeiramente \\
( ) Indiferente \\
( ) Desgostei ligeiramente \\
( ) Desgostei moderadamente \\
( ) Desgostei muito \\
( ) Desgostei extremamente
\end{tabular}

\subsection{MAPA DE PREFERÊNCIA INTERNO}

Para obtenção do Mapa de Preferência Interno, os dados de aceitação foram organizados numa matriz de amostras (em linhas) e consumidores (em colunas). Os dados foram submetidos à Análise de Componentes Principais (ACP) a partir da matriz de covariâncias.

Os resultados foram expressos em gráficos de dispersão das amostras (tratamentos) em relação aos dois primeiros componentes principais e outro representando as correlações dos dados de cada consumidor com os escores dos dois primeiros componentes principais.

\section{RESULTADOS E DISCUSSÃO}

\subsection{ANÁLISE DESCRITIVA QUANTITATIVA}

Após consenso dos julgadores sobre os termos que melhor caracterizavam as amostras (Quadro 1) foi elaborada para a composição da ficha de avaliação das amostras, a definição dos oito termos descritivos e a dos termos extremos das escalas nãoestruturadas. 


\section{QUADRO 1- DEFINIÇÃO DOS ATRIBUTOS SENSORIAIS DO REFRIGERANTE SABOR GUARANÁ E AMOSTRAS DE REFERÊNCIA}

\begin{tabular}{|c|c|c|}
\hline Atributos & Defini I es & ReferCrcias \\
\hline Cor caramelo & \begin{tabular}{|lr} 
Cor & caramelo \\
caracter stica & de \\
refrigerante & sabor \\
guaran $Æ E$ & \\
\end{tabular} & $\begin{array}{l}\text { Fraca: } 100 \mathrm{~mL} \text { de refrigerante da marca } \\
\text { Americana }+30 \mathrm{~mL} \text { de Agua destilada } \\
\text { Forte: } 100 \mathrm{~mL} \text { de refrigerante da marca } \\
\text { Americana }+30 \mathrm{~mL} \text { de caramelo }\end{array}$ \\
\hline $\begin{array}{l}\text { Aroma e sabor } \\
\text { caracter sticos }\end{array}$ & \begin{tabular}{|lr} 
Aroma e & sabor \\
caracter sticos & de \\
refrigerante & sabor \\
guaran $Æ$ &
\end{tabular} & $\begin{array}{l}\text { Pouco: refrigerante da mar } \\
\text { Muito: refrigerante da marc }\end{array}$ \\
\hline $\begin{array}{l}\text { Aroma e sabor } \\
\text { tutti-fruti }\end{array}$ & $\begin{array}{lr}\text { Aroma e r } & \text { sabor } \\
\text { associados } & \text { mistura } \\
\text { de frutas } & \end{array}$ & $\begin{array}{l}\text { Ausente: refrigerante da marca Antarctica } \\
\text { Muito: } 100 \mathrm{~mL} \text { de refrigerante da marca } \\
\text { Ver }-0+3 \text { gotas de aroma de banana }+2 \\
\text { gotas de aroma de morango }\end{array}$ \\
\hline EfervescCF́cia & $\begin{array}{l}\text { Sensa o associada } \\
\text { presen a de g/ts } \\
\text { durante a degusta }{ }^{a} \text { o }\end{array}$ & $\begin{array}{l}\text { Pouca: refrigerante da marca Antarctica } \\
\text { com pouco g/ts } \\
\text { Muita: refrigerante da marca Antarctica }\end{array}$ \\
\hline Doce & $\begin{array}{l}\text { Gosto associado } \\
\text { solu a o de sacarose }\end{array}$ & $\begin{array}{l}\text { Pouco: } 100 \mathrm{~mL} \text { de refrigerante da marca } \\
\text { Antarctica }+30 \mathrm{~mL} \text { de } \text { Ægua destilada } \\
\text { Muito: } 100 \mathrm{~mL} \text { de refrigerante da marca } \\
\text { Picolino }+2 \text { gramas de sacarose }\end{array}$ \\
\hline Medicinal & $\begin{array}{lr}\text { Sabor } & \text { associado } \\
\text { xarope } & \text { infantil } \\
\text { (remødio) } & \end{array}$ & $\begin{array}{l}\text { Ausente: refrigerante da marca Antarctica } \\
\text { Muito: refrigerante da marca Fanny }\end{array}$ \\
\hline Frutas c tricas & $\begin{array}{l}\text { Sabor } \\
\text { solu associado } \\
\text { c trico de }\end{array}$ & $\begin{array}{l}\text { Ausente: refrigerante da marca Antarctica } \\
\text { Muito: } 100 \mathrm{~mL} \text { de refrigerante da marca } \\
\text { Pet Mil }+0,2 \text { gramas de Feido } \mathrm{c} \text { trico }\end{array}$ \\
\hline Ado & $\begin{array}{|ll|}\text { Sabor } & \text { residual } \\
\text { associado } & \text { solu } \stackrel{a}{0} 0 \\
\text { de sacarina } & \end{array}$ & $\begin{array}{l}\text { Ausente: refrigerante da marca Antarctica } \\
\text { Muito: } 100 \mathrm{~mL} \text { de refrigerante da marca } \\
\text { Fanny }+8 \text { gotas de ado ante (Assugrin) }\end{array}$ \\
\hline
\end{tabular}

As amostras diferiram significativamente $(p \leq 0,05)$ entre si em todos os atributos avaliados, exceto para o gosto doce (Tabela 1). A interação amostra $X$ julgador foi significativa $(p \leq 0,05)$ para os atributos aroma característico, aroma tutti-fruti, efervescência e sabores doce, medicinal e adoçante. Desta forma, o teste para efeito de tratamento foi realizado conforme STONE e SIDEL (1993), utilizando o quadrado médio da interação.

A Figura 2 apresenta os resultados da ACP dos atributos sensoriais para as amostras de refrigerante. Os dois primeiros componentes principais explicaram $83 \%$ da variação total entre as amostras quanto a seus atributos sensoriais, sendo que o primeiro explicou $64 \%$ e o segundo $19 \%$. Assim, apenas os dois primeiros componentes principais foram suficientes para discriminar as amostras. 
TABELA 1-RESUMO DA ANÁLISE DE VARIÂNCIA DOS ATRIBUTOS SENSORIAIS DE REFRIGERANTE

\begin{tabular}{|c|c|c|c|c|c|c|c|}
\hline \multirow[b]{2}{*}{ Atributo } & \multirow[b]{2}{*}{ FV } & \multirow[b]{2}{*}{ GL } & \multirow[b]{2}{*}{ QM } & \multicolumn{2}{|c|}{ versus res duo } & \multicolumn{2}{|c|}{ versus intera ${ }^{a} 0$} \\
\hline & & & & $\mathbf{F}$ & Prob. & $\mathbf{F}$ & Prob. \\
\hline & $A$ & 6 & 84,53 & 44,01 & $<0.0001$ & & \\
\hline \multirow{3}{*}{ Cor Caramelo } & J & 8 & 4,18 & 2,17 & 0.0336 & & \\
\hline & AxJ & 48 & 0,99 & 0,52 & 0.9944 & & \\
\hline & Res duo & 126 & 1,92 & & & & \\
\hline & A & 6 & 116,70 & 32,74 & $<0.0001$ & 21,72 & $<0.0001$ \\
\hline Aroma & J & 8 & 28,36 & 7,96 & $<0.0001$ & & \\
\hline \multirow[t]{2}{*}{ caracter stico } & AxJ & 48 & 5,37 & 1,51 & 0.0368 & & \\
\hline & Res duo & 126 & 3,56 & & & & \\
\hline \multirow{4}{*}{$\begin{array}{c}\text { Aroma Tutti- } \\
\text { fruti }\end{array}$} & A & 6 & 49,01 & 14,63 & $<0.0001$ & 6,29 & $<0.0001$ \\
\hline & $J$ & 8 & 22,71 & 6,78 & $<0.0001$ & & \\
\hline & AxJ & 48 & 7,79 & 2,32 & $<0.0001$ & & \\
\hline & Res duo & 126 & 3,35 & & & & \\
\hline \multirow{4}{*}{$\begin{array}{c}\text { Sabor } \\
\text { caracter stico }\end{array}$} & $A$ & 6 & 125,53 & 38,28 & $<0.0001$ & & \\
\hline & $J$ & 8 & 11,17 & 3,40 & 0.0014 & & \\
\hline & AxJ & 48 & 4,55 & 1,39 & 0.0765 & & \\
\hline & Res duo & 126 & 3,28 & & & & \\
\hline \multirow{4}{*}{ EfervescC̄cia } & A & 6 & 12,53 & 4,45 & 0.0004 & 2,62 & 0.0284 \\
\hline & $J$ & 8 & 46,56 & 16,53 & $<0.0001$ & & \\
\hline & AxJ & 48 & 4,79 & 1,70 & 0.0101 & & \\
\hline & Res duo & 126 & 2,82 & & & & \\
\hline \multirow{4}{*}{ Doce } & $A$ & 6 & 17,74 & 5,63 & $<0.0001$ & 2,23 & 0.0561 \\
\hline & $J$ & 8 & 27,12 & 8,61 & $<0.0001$ & & \\
\hline & $A x J$ & 48 & 7,96 & 2,53 & $<0.0001$ & & \\
\hline & Res duo & 126 & 3,15 & & & & \\
\hline \multirow{4}{*}{ Medicinal } & A & 6 & 58,51 & 14,85 & $<0.0001$ & 6,69 & $<0.0001$ \\
\hline & J & 8 & 48,31 & 12,27 & $<0.0001$ & & \\
\hline & $A x J$ & 48 & 8,75 & 2,22 & 0.0002 & & \\
\hline & Res duo & 126 & 3,94 & & & & \\
\hline \multirow{4}{*}{$\begin{array}{l}\text { Frutas } \\
\text { c tricas }\end{array}$} & $A$ & 6 & 18,16 & 3,99 & 0.0011 & & \\
\hline & $\mathrm{J}$ & 8 & 48,20 & 10,60 & $<0.0001$ & & \\
\hline & AxJ & 48 & 6,17 & 1,36 & 0.0915 & & \\
\hline & Res duo & 126 & 4,55 & & & & \\
\hline \multirow{4}{*}{ Tutti-fruti } & $A$ & 6 & 27,58 & 7,64 & $<0.0001$ & & \\
\hline & J & 8 & 56,70 & 15,71 & $<0.0001$ & & \\
\hline & $A x J$ & 48 & 6,34 & 1,76 & 0.0069 & & \\
\hline & Res duo & 126 & 3,61 & & & & \\
\hline \multirow{4}{*}{ Ado ante } & $A$ & 6 & 57,12 & 14,74 & $<0.0001$ & 10,03 & $<0.0001$ \\
\hline & J & 8 & 24,63 & 6,36 & $<0.0001$ & & \\
\hline & AxJ & 48 & 5,69 & 1,47 & 0.0468 & & \\
\hline & Res duo & 126 & 3,88 & & & & \\
\hline
\end{tabular}

$A=$ amostra; $\mathrm{J}=$ julgador e $\mathrm{AxJ}=$ interação amostra versus julgador. 
FIGURA 2 - RESULTADOS OBTIDOS PELA ANÁLISE DE COMPONENTES PRINCIPAIS DOS ATRIBUTOS SENSORIAIS PARA AS AMOSTRAS DE REFRIGERANTE (A E B)
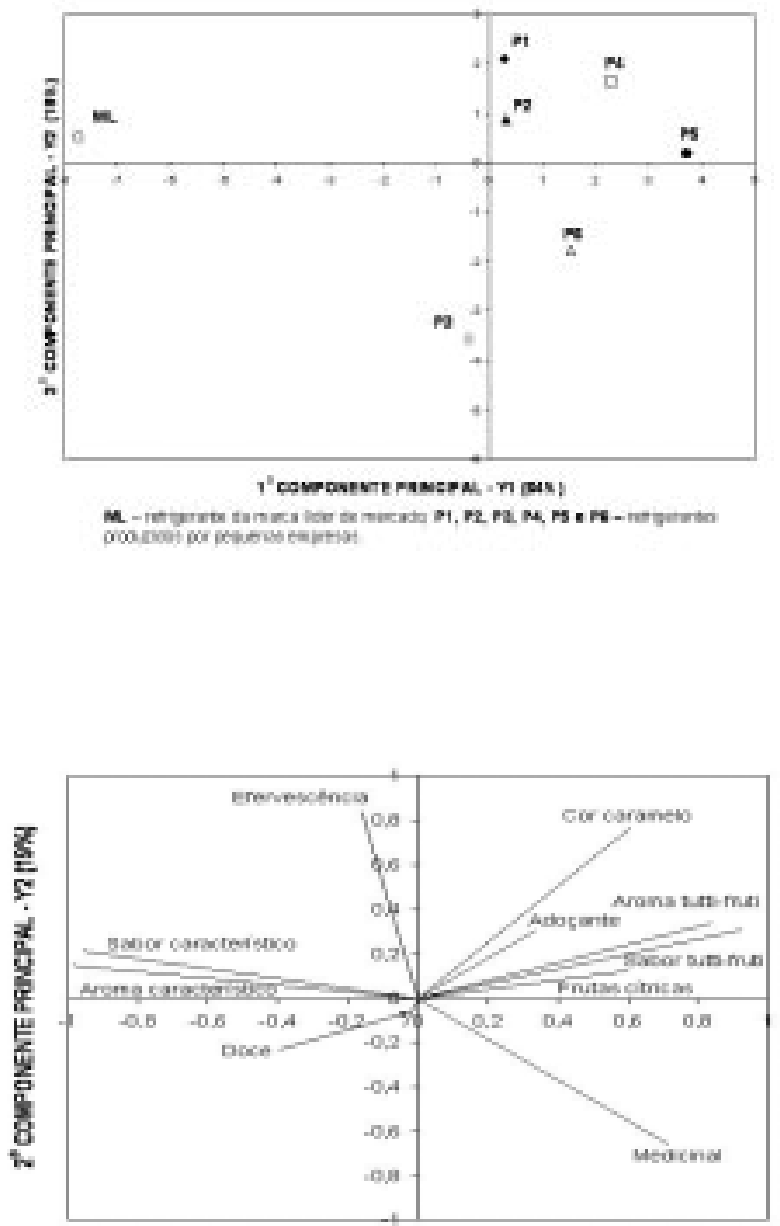

1 " COMPONENTE PRINEPAL - Y1 Masa 
A separação espacial das sete marcas de refrigerante indica que o da marca líder diferiu dos refrigerantes produzidos por pequenas empresas (Figura 2A).

Os atributos aroma e sabor característicos e aroma e sabor tutti-fruti apresentaram correlação significativa com o primeiro componente principal ( $r \geq 0,84$ em valor absoluto) ao nível de 5\% de significância. Logo, esses atributos discriminaram as amostras em relação a esse componente (Tabela 2).

\section{TABELA 2 - COEFICIENTES DE CORRELAÇÃO DE PEARSON ENTRE OS ATRIBUTOS SENSORIAIS E OS DOIS PRIMEIROS COMPONENTES PRINCIPAIS}

\begin{tabular}{|c|c|c|c|c|}
\hline \multirow{3}{*}{ ATRIBUTOS } & \multicolumn{4}{|c|}{ Componentes Principais } \\
\hline & \multicolumn{2}{|c|}{ Y1 } & \multicolumn{2}{|c|}{ Y2 } \\
\hline & $r$ & $p$ & $r$ & $p$ \\
\hline Cor caramelo & $0.61^{\star \star}$ & 0.14 & $0.77^{*}$ & 0.04 \\
\hline Aroma Caracter stico & $-0.99^{*}$ & 0.00 & 0.15 & 0.76 \\
\hline Aroma Tutti-fruti & $0.84^{*}$ & 0.02 & 0.34 & 0.46 \\
\hline Sabor Caracter stico & $-0.96^{*}$ & 0.00 & 0.21 & 0.66 \\
\hline EfervescCfrcia & -0.16 & 0.73 & $0.84^{*}$ & 0.02 \\
\hline Doce & -0.04 & 0.94 & -0.13 & 0.79 \\
\hline Medicinal & $0.72^{\star \star}$ & 0.07 & $-0.66^{\star \star}$ & 0.11 \\
\hline Frutas c tricas & 0.61 & 0.16 & 0.12 & 0.81 \\
\hline Tutti-fruti & $0.93^{*}$ & 0.00 & 0.31 & 0.50 \\
\hline Ado ante & 0.34 & 0.46 & 0.31 & 0.50 \\
\hline
\end{tabular}

$r$ = Coeficiente de correlação de Pearson; $p=$ nível de significância.

${ }^{*}$ correlação significativa $(p \leq 0.05) ;{ }^{* *}$ correlação significativa $(p \leq 0.15)$.

Os resultados apresentados na Tabela 2 e Figura 2B sugerem que os atributos aroma e sabor característicos $(r \leq-0,96)$ estão presentes em maior intensidade no refrigerante ML. Já os atributos aroma e sabor tutti-fruti $(r \geq 0,84)$, cor caramelo e sabor medicinal $(r \geq 0,61)$ estão presentes em maior intensidade nos refrigerantes produzidos por pequenas empresas (correlacionados com o primeiro componente principal).

Comparando os refrigerantes produzidos por pequenas empresas observou-se menor intensidade dos atributos cor caramelo $(r \geq 0,77)$ e efervescência $(r \geq 0,84)$ na amostra $P 3$, devido a correlação positiva com o segundo componente principal. 


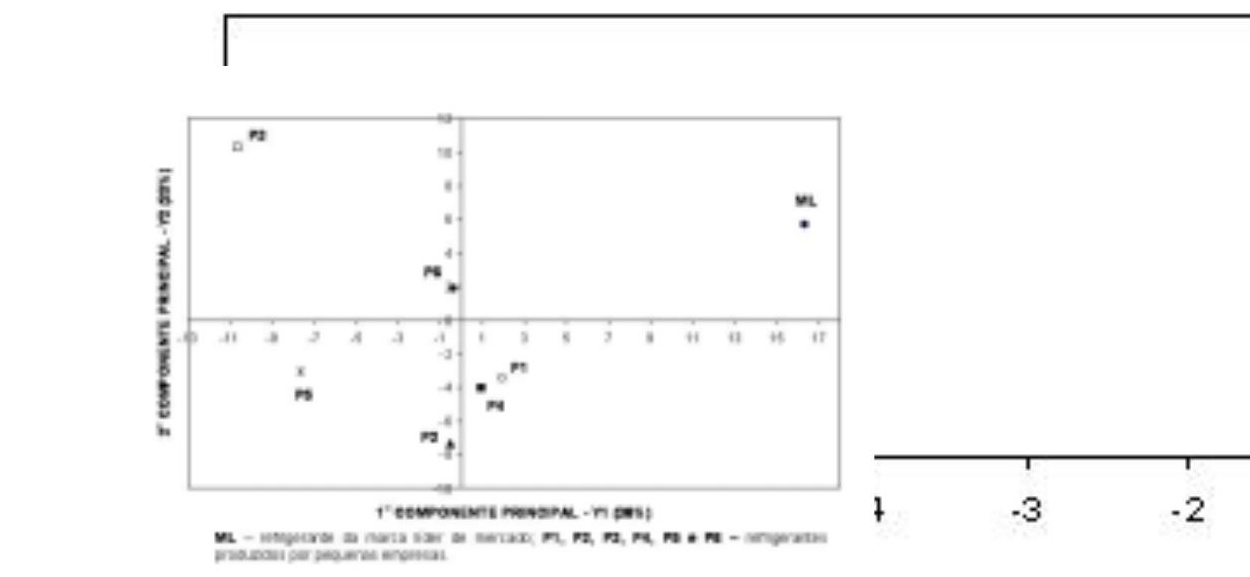

A - Dispersãe-as|amostras de refrigerante em relação à aceitação pelos consumidores

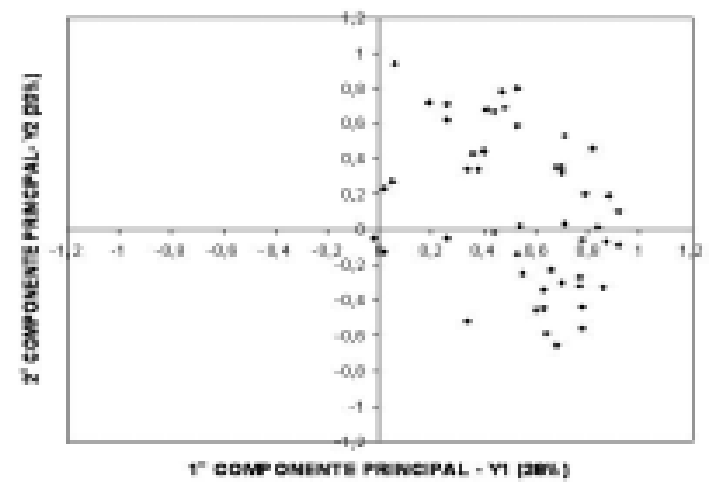

B - Correlaçõels entre os dados de aceitação de cada consumidor e os dois primeiros componentes principais

\section{$1{ }^{\circ}$ COMPONENTE $\mathrm{P}$}

$\mathbf{M} \mathbf{L}$ - refrigerante da marca líder de merca produzidos por pecuenas empresas 
A separação espacial das amostras de refrigerante indica diferença na aceitação das mesmas (Figura 3-A). O refrigerante da marca líder $(\mathrm{ML})$ diferiu dos demais. Comparando os refrigerantes produzidos por pequenas empresas observou-se que P1, P2, P4 e P6 são os mais semelhantes, diferindo de P3 e P5 (discriminação clara em relação ao primeiro componente principal).

Na Figura 3-B cada ponto representa a correlação entre os dados de aceitação de um consumidor e os dois primeiros componentes principais. A correlação entre consumidores com pelo menos um dos componentes indica diferença na aceitação das amostras. A maioria dos consumidores (64\%) preferiu o refrigerante da marca líder $(\mathrm{ML})$, verificando-se correlação positiva $(r \geq 0,50)$ com o primeiro componente principal.

Em relação ao segundo componente principal também foi observada correlação positiva $(r \geq 0,50)$, com $22 \%$ dos consumidores preferindo ML e P3 e correlação negativa $(r \leq-0,50)$, com $8 \%$ preferindo a P2.

\section{CONCLUSÃO}

Os atributos que contribuíram para a diferenciação das amostras avaliadas foram aroma e sabor característico, aroma e sabor tutti-fruti, cor caramelo e sabor medicinal. O refrigerante da marca líder (ML) apresentou os atributos aroma e sabor característico em maior intensidade, diferindo dos refrigerantes produzidos por pequenas empresas que revelaram maior intensidade para os atributos aroma e sabor tutti-fruti, cor caramelo e sabor medicinal.

O refrigerante da marca líder ( $\mathrm{ML}$ ) também diferiu dos refrigerantes produzidos por pequenas empresas quanto à aceitação, sendo preferido por $64 \%$ dos consumidores. Comparando-se os refrigerantes produzidos por pequenas empresas observou-se que P1, P2, P4 e P6 são semelhantes, diferindo de P3 e P5. Em relação ao segundo componente principal, $22 \%$ dos consumidores preferiram as amostras ML e P3 e $8 \%$ a P2. 
A - Dispersão das amostras de refrigerante em relação aos dois primeiros componentes principais.

B - Correlações entre os atributos sensoriais e os dois primeiros componentes principais.

3.2 MAPA DE PREFERÊNCIA INTERNO

No Mapa de Preferência Interno (Figura 3), o primeiro componente explicou 38\% da variação de aceitação entre as amostras de refrigerante e o segundo $20 \%$. Os dois primeiros componentes explicaram $58 \%$ da variância entre as amostras quanto à sua aceitação.

\section{FIGURA 3 - MAPA DE PREFERÊNCIA INTERNO PARA AS AMOSTRAS DE REFRIGERANTE (A E B)}


9 SEAE. Secretaria de Acompanhamento Econômico. Parecer sobre Ato Concentração n. 08012.005846/99-12 (AMBEV). Revista do Ibrac (Instituto Brasileiro de Estudos das Relações de Concorrência e de Consumo), Doutrina, Jurisprudência e Legislação, v.7, n.3, 2000.

10 STONE, H., SIDEL, J. L., OLIVER, S., WOOLSEY, A., SINGLETON, R.C. Sensory evaluation by quantitative descriptive analysis. Food Technology, v.28, n.11, p.24-34, 1974.

11 STONE, H.; SIDEL, J.L. Sensory evaluation: practices. $2^{\text {nd }}$ ed. London: Academic Press, 1993. $337 \mathrm{p}$. 